\title{
High-Resolution Crystal Structure of the Snake Venom Metalloproteinase BaP1 Complexed with a Peptidomimetic: Insight into Inhibitor Binding:
}

\author{
Torsten Lingott, ${ }^{\S}$ Christian Schleberger, ${ }^{\S}$ José María Gutiérrez," and Irmgard Merfort*\$ \\ ${ }^{\S}$ Department of Pharmaceutical Biology and Biotechnology, Institute of Pharmaceutical Sciences, Albert-Ludwigs-Universität, \\ Stefan-Meier-Strasse 19, 79104 Freiburg im Breisgau, Germany, and "Clodomiro Picado Institute, Faculty of Microbiology, \\ University of Costa Rica, San José, Costa Rica
}

Received February 11, 2009; Revised Manuscript Received May 28, 2009

\begin{abstract}
BaP1, a zinc-dependent endopeptidase belonging to the P-I class of snake venom metalloproteinases, exerts multiple tissue-damaging activities, leading to hemorrhage, myonecrosis, dermonecrosis, blistering, and edema. Interestingly, this metalloproteinase shows a high degree of structural homology with the catalytic domain of human adamalysins and matrix metalloproteinases, especially at the strictly conserved zinc binding motif and the so-called Met turn. This highlights $\mathrm{BaP} 1$ as an interesting model concerning inhibitor design for several medicinally important metalloproteinases, such as tumor necrosis factor $\alpha$ converting enzyme. Here, we report the first crystal structure of BaP1 complexed with a peptidomimetic inhibitor. Suitable crystals were obtained at four different $\mathrm{pH}$ values $(4.6,6.5,7.5$, and 8.0$)$, and four highresolution structures $(1.46,1.14,1.08$, and $1.05 \AA$ ) were established. These structures and the detailed analysis of the structure-activity relationship of the bound inhibitor form a basis for the design of potent BaP1 inhibitors. The latter can be used for the treatment of local pathological effects caused by snake bites, mainly due to metalloproteinases such as BaP1. Besides, the high-resolution structure is an excellent starting point for the rational development of inhibitors for human metalloproteinases. The finding of a flexible loop region may have a great impact on further studies as to date little is known about the structural dependencies of the hemorrhagic activity of snake venom metalloproteinases.
\end{abstract}

Metalloendopeptidases (MEPs) ${ }^{1}$ from snake venoms [snake venom metalloproteinases (SVMPs)] and ADAMs (a disintegrin and metalloproteinase domain-containing enzymes) constitute the reprolysin subfamily of the "metzincin" superfamily of zincdependent metalloendopeptidases ( 1 ). Metzincins have two characteristic features: the conserved zinc binding motif HEbxHxbGbxHD (b, bulky hydrophobic residue; $\mathrm{x}$, any residue) that contains the catalytic glutamate residue and the so-called Met turn, a methionine-containing 1,4- $\beta$-turn that plays an important structural role near the active site $(2-6)$. A reaction mechanism in which the catalytic glutamate polarizes a zincbound water molecule, thereby increasing its nucleophilic character, has been proposed. The polarized water subsequently cleaves the substrate peptide bond in an addition-elimination mechanism $(3,4,7)$. Efforts are being made to investigate the $\mathrm{pH}$

\footnotetext{
Coordinates and structure factors of the $\mathrm{BaP} 1 \cdot$ inhibitor complex have been deposited at the Protein Data Bank as entries 2W12, 2W13, $2 \mathrm{~W} 14$, and $2 \mathrm{~W} 15$.

*To whom correspondence should be addressed. E-mail: irmgard. merfort@pharmazie.uni-freiburg.de. Phone: 0049-(0)761-203-8373. Fax: 0049-(0)-761-203-8383.

${ }^{1}$ Abbreviations: MEPs, metalloendopeptidases; SVMPs, snake venom metalloproteinases; ADAMs, a disintegrin and metalloproteinasecontaining enzymes; MMPs, matrix metalloproteinases; TACE, tumor necrosis factor $\alpha$ converting enzyme; TNF- $\alpha$, tumor necrosis factor $\alpha$; XANES, X-ray absorption near edge spectroscopy; rms, root-meansquare; ZBG, zinc binding group.
}

dependency of the proteolytic activity of SVMPs and other metzincins, generally known to be higher in the neutral to basic range (4). It was assumed that at a lower $\mathrm{pH}$, the capacity of the glutamate residue to polarize the catalytic water molecule is diminished, preventing nucleophilic attack on the scissile peptide bond.

After the first ADAM was discovered in guinea pig sperm, it became clear that these enzymes possess important biological properties and that the catalytic domains of ADAMs and SVMPs are structurally very similar, particularly in the fold and the arrangement of the active site cleft $(3,8-10)$. ADAMs have been implicated in a set of diseases, i.e., diabetes, Alzheimer's disease, cardiac hypertrophy, cancer, rheumatoid arthritis, Crohn's disease, and microbial infections $(8,11)$. Especially ADAM-17, also known as TACE (tumor necrosis factor $\alpha$ converting enzyme), deserves high interest as a promising medicinal target, as it cleaves the membrane-bound precursor of tumor necrosis factor $\alpha(\mathrm{TNF}-\alpha)$ to its soluble form $(12,13)$.

Every year more than 2.6 million people worldwide are envenomed by snake bites, leading to more than 125000 deaths (14). Snake venoms are a complex mixture of proteins, inorganic compounds, and metal ions (4). While systemic pathophysiological alterations are readily avoided by timely administration of antivenoms, local pathological effects are difficult to neutralize, primarily because of the very rapid development of these lesions (15). The main venom components responsible for 
local pathology are myotoxic phospholipases $\mathrm{A}_{2}$ and metalloproteinases (16). In particular, the zinc-dependent SVMPs cause hemorrhagic effects, myonecrosis, skin damage, edema, and other reactions associated with inflammation as well as relevant hemostatic and hematological alterations (17-19). Several SVMPs have been characterized, and they are presently categorized into three main classes (P-I, P-II, and P-III) based on their different sizes and domain structures $(1,17,20)$. One of these SVMPs is BaP1, isolated from the venom of Bothrops asper, the medically most important snake in Central America (21). It is a 22.7 kDa P-I SVMP exhibiting high proteolytic activity and locally inducing hemorrhage, inflammation, and moderate myonecrosis. After intramuscular injection, it causes formation of blisters, infiltration of leukocytes, and the release of cytokines, including interleukin-1 and TNF- $\alpha(22,23)$. The maximum proteolytic activity of $\mathrm{BaP} 1$ in vitro occurs at $\mathrm{pH} 8.0$, whereas the physiological reaction conditions are around $\mathrm{pH} 7.4$ (18).

One of the main features of viperid snake venom is the ability to induce hemorrhage in local tissue, which is mainly due to SVMPs $(16,17,23)$. The intensity of hemorrhagic activity varies greatly between the different classes of SVMPs, with the P-III class usually being more hemorrhagic than the P-I class. This was explained by the existence of additional domains, better access to physiologically relevant targets in microvessels, and the abillity of P-III SVMPs to inhibit collagen-induced platelet aggregation (24). However, there are also large differences in hemorrhagic activity within the P-I class of SVMPs, indicating that structural features in the metalloproteinase domain itself may play a role. Comparisons of several SVMPs with different hemorrhagic potential have shown that there are conspicuous structural variations in a loop region surrounding the active site $(24,25)$. Recently, a bioinformatics study involving comparative surface analysis of several hemorrhagic and nonhemorrhagic SVMPs (P-I as well as P-III) revealed small differences in molecular surface area within the P-I SVMPs that correlate with hemorrhagic potential (26).

Although long appreciated in traditional practice, the potential of snake venoms and their components as a basis for drug design and novel therapies has only recently been recognized by modern medicine $(27,28)$. One of the first examples was the development of synthetic inhibitors for angiotensin converting enzyme using the structure of an oligopeptide from Bothrops jararaca venom (29). Structural similarities between snake venom proteins and human enzymes offer intriguing possibilities for the development of inhibiting compounds. Accordingly, several SVMP-inhibitor/substrate complexes have already been used to investigate structure-activity relationships and to design inhibitors for metalloproteinases (4, 30-35). Different zinc binding moieties are known that can be used to guide these inhibitors to the active site of metzincins. Among these, hydroxamate derivatives with peptidomimetic scaffolds exhibit an especially high potential and are therefore of great interest for the development of small molecule inhibitors for SVMPs, ADAMs, and MMPs (36).

The overall structure of BaP1 [Protein Data Bank (PDB) entry 1ND1] (24) is very similar to the seven other structurally known P-I SVMPs $(7,25,34,35,37-40)$. It consists of a major Nterminal subdomain (residues 1-152) and a minor C-terminal subdomain (residues 153-202) which flank the shallow active site cleft. The major domain adopts an $\alpha / \beta$-fold containing four $\alpha$-helices $(\alpha 1, \alpha 2, \alpha 3$, and $\alpha 4)$ and a five stranded $\beta$-sheet $(\beta 1, \beta 2, \beta 3, \beta 4$, and $\beta 5)$. All strands are parallel except antiparallel strand $\beta 4$. The minor domain consists of one $\alpha$-helix $(\alpha 5)$ and various loops, including the conserved Met turn.

Here, the first crystal structure of the SVMP BaP1 in complex with a metalloproteinase inhibitor, primarily used as a TACE inhibitor, is reported. To structurally show the dependency of the proteolytic activity on the $\mathrm{pH}$ value, the crystallization procedure was carried out at different $\mathrm{pH}$ values. Suitable crystals were obtained at four different $\mathrm{pH}$ values $(4.6,6.5,7.5$, and 8.0), and four high-resolution structures (1.46, 1.14, 1.08, and 1.05 $\AA$ ) were established. This structural analysis offers new insight into the $\mathrm{pH}$ dependency of inhibitor binding as well as important structural and functional aspects of a flexible loop region linked to hemorrhagic activity. In addition, this study improves our understanding of metalloproteinases and forms a solid platform for the rational design of inhibitors for metzincins like BaP1, TACE, or MMPs.

\section{EXPERIMENTAL PROCEDURES}

Protein Purification. BaP1 was isolated from a venom pool collected from adult specimens of $B$. asper of the Pacific regions of Costa Rica. After extraction, venom was lyophilized and stored at $-20{ }^{\circ} \mathrm{C}$. BaPl was purified following established methods $(18,19)$. Briefly, initial purification was done via ionexchange chromatography on a CM Sephadex C-25 column (Pharmacia), followed by an affinity chromatography on Affi Gel Blue column material (Bio-Rad). After BaP1 was judged to be pure by SDS-PAGE (41), it was either lyophilized or directly used for assays. For crystallization experiments, lyophilized BaP1 was diluted in buffer A $[15 \mathrm{mM}$ Tris- $\mathrm{HCl}(\mathrm{pH} 8.0)$ and $200 \mathrm{mM}$ sodium chloride] and loaded onto a Superdex-200 26/60 column (Amersham Biosciences) preequilibrated with buffer A. BaP1containing fractions were pooled, concentrated, and either directly used for crystallization experiments or cryocooled in liquid nitrogen and stored at $-80{ }^{\circ} \mathrm{C}$.

Protease Inhibition Assays. The proteolytic activity of $\mathrm{BaP} 1$ was assayed by unselective cleavage of azocasein (42). Different concentrations of purified BaP1 were diluted in $20 \mu \mathrm{L}$ of buffer B [25 mM Tris- $\mathrm{HCl}$ (pH 7.4), $150 \mathrm{mM}$ sodium chloride, and $5 \mathrm{mM}$ calcium chloride] and were mixed with $100 \mu \mathrm{L}$ of a solution of 10 $\mathrm{mg} / \mathrm{mL}$ azocasein in buffer B. After an incubation for $90 \mathrm{~min}$ at $37^{\circ} \mathrm{C}, 200 \mu \mathrm{L}$ of $5 \%$ (v/v) trichloroacetic acid was added to quench the reaction and the samples were centrifuged for $10 \mathrm{~min}$ at $4000 \mathrm{~g} ; 100 \mu \mathrm{L}$ of the supernatant was mixed with an equal amount of $0.5 \mathrm{M}$ sodium hydroxide, and the absorbance at 450 $\mathrm{nm}$ was recorded. For inhibition testing, BaP1 $(0.7 \mathrm{mg} / \mathrm{mL})$ was preincubated for $30 \mathrm{~min}$ at $37^{\circ} \mathrm{C}$ with different concentrations of the inhibitor in buffer $\mathrm{B}$ before addition of the azocasein solution. Assays were performed in triplicate. Statistical analysis and nonlinear regression were performed using Prism 5.0 (GraphPad Software, Inc.).

Protein Crystallization. Crystals of $\mathrm{BaP} 1$ were obtained via hanging-drop vapor diffusion at $21{ }^{\circ} \mathrm{C}$. BaPl-inhibitor complexes at $\mathrm{pH} 4.6,6.5$, and 7.5 were obtained by mixing equal volumes of a protein solution $(5-10 \mathrm{mg} / \mathrm{mL})$ containing $880 \mu \mathrm{M}$ inhibitor with the following reservoir solutions: crystal I ( $\mathrm{pH} 4.6)$, $100 \mathrm{mM}$ sodium acetate, $30 \%(\mathrm{w} / \mathrm{v})$ polyethylene glycol 4000 , and $200 \mathrm{mM}$ ammonium acetate; crystal II (pH 6.5), $100 \mathrm{mM}$ Tris and $30 \%(\mathrm{w} / \mathrm{v})$ polyethylene glycol 20000; crystal III ( $\mathrm{pH} 7.5)$, $100 \mathrm{mM}$ HEPES, 20\% (w/v) polyethylene glycol 3000, and 200 $\mathrm{mM}$ sodium chloride. Crystals grew within a few days as thin plates, all belonging to orthorhombic space group $P 2_{1} 2_{1} 2_{1}$. 
To obtain the inhibitor complex at $\mathrm{pH} 8.0$ (crystal IV), a native crystal grown in $100 \mathrm{mM}$ imidazole $(\mathrm{pH} 8.0)$ and $10 \%(\mathrm{w} / \mathrm{v})$ polyethylene glycol 8000 was placed into a drop containing 3.0 $\mathrm{mg} / \mathrm{mL} \mathrm{BaP} 1$ and $550 \mu \mathrm{M}$ inhibitor in the same buffer and equilibrated for $70 \mathrm{~h}$. All crystals were cryoprotected in their respective reservoir solutions containing $10 \%(\mathrm{v} / \mathrm{v})$ glycerol and flash-frozen in liquid nitrogen.

Data Collection, Model Building, and Refinement. Native data sets were collected at $100 \mathrm{~K}$ and a wavelength of $0.92 \AA$ at beamline BL14.1 at BESSY (Berliner ElektronenspeicherringGesellschaft fur Synchrotronstrahlung m.b.H., Berlin, Germany) (Table 1). Diffraction data were processed and scaled using XDS and XSCALE (43). Because the BaP1 -inhibitor crystals in this study were isomorphous to previously reported native $\mathrm{BaP} 1$ crystals (24), the structures of the inhibitor complexes were determined using difference Fourier methods. Initial electron density maps were obtained after rigid body refinement with REFMAC (44) using the unliganded BaP1 structure (PDB entry 1ND1) (24) as a starting model. After rebuilding with COOT (45) and refinement with REFMAC, the inhibitors were modeled into the resulting $\left(F_{\mathrm{o}}-F_{\mathrm{c}}\right)$ difference electron density maps. An energy-minimized conformation of the inhibitor and the corresponding dictionary files were generated with PRODRG2 (46). Water molecules were placed with ARP/wARP (47) and checked with COOT. The model was refined using isotropic temperature factors for data set II and anisotropic temperature factors for the other data sets (Table 2). The atoms of the loop region between residues 159 and 162 were modeled with half-occupancy concerning to the two conformations of the loop. All figures were produced with MOLSCRIPT and POVScript $+(48,49)$.

\section{RESULTS AND DISCUSSION}

Crystals of $\mathrm{BaP} 1$ in complex with a hydroxamate inhibitor were obtained at four different $\mathrm{pH}$ values, and the structures were determined to atomic resolution (Tables 1 and 2). The structures of the inhibitor complex were determined by difference Fourier methods using the structure of native BaP1 (24). Because there were no significant conformational changes between the four structures, the following discussion will refer to the $1.08 \AA$ structure obtained at $\mathrm{pH} 8.0$ (data set IV), unless stated otherwise. During structure analysis, it became clear that residue 151, originally reported to be an aspartate due to poor electron density, is in fact a histidine.

In the $\mathrm{BaP} 1$ - inhibitor structure, all residues are well-defined in the electron density, with the exception of a few amino acids with disordered side chains at the protein surface. The electron density for the inhibitor was continuous at the $3 \sigma$ contour level and allowed unambiguous placement of its scaffold (Figure 1A). In all four crystal structures, an additional glycerol molecule from the cryoprotectant was identified near the active site, which is held into place by hydrogen bonds to the $\mathrm{N} \varepsilon$ atom of $\operatorname{Arg} 110$, a water molecule, and the inhibitor. A loop segment containing residues 159-162 existed in a double conformation in all four crystals and was modeled as such.

Interaction between BaPl and the Inhibitor. The synthetic hydroxamate derivative used in this study inhibited $\mathrm{BaP} 1$ with an $\mathrm{IC}_{50}$ value of $22.2 \mu \mathrm{M}$ (Figure 2). This peptidomimetic was originally designed as a TACE inhibitor (personal communication, Roche Pharmaceutics). Due to the fact that TACE and $\mathrm{BaP} 1$ have structurally very similar binding sites, it was then used to analyze its effects on snake venom metalloproteinases. In the structure, the hydroxamate derivative occupies the peptide binding cleft of $\mathrm{BaP} 1$, forming interactions over its entire length. As points of reference, the peptidomimetic inhibitor can be separated into four parts (Figure 3). In the following, functional groups, namely, hydroxamate (Hyd1), isobutyl (Byl2), tert-butyl (Byl3), and methyl (Myl4), are used to identify the different parts of the inhibitor. The N-methylthiazole-2-carboxamide group of Byl2 is shortened to thiazole. Following common nomenclature for peptide/protease complexes, these groups are divided into $\mathrm{P} 1$ (thiazole), $\mathrm{P}^{\prime}$ (Byl2), $\mathrm{P}^{\prime}$ (Byl3), and P3' (Myl4). In its binding mode, the inhibitor closely mimics the C-terminal part of an enzyme-bound peptide substrate. It is held in position by hydrogen bonds and van der Waals contacts involving its backbone and side chains as well as a cation $-\pi$ interaction (Figure 4). The hydrogen bonding network between the enzyme and inhibitor backbone resembles that of an antiparallel $\beta$-sheet.

Four regions of $\mathrm{BaP} 1$ are involved in inhibitor binding. The first consists of part of strand $\beta 4$ and the proceeding loop. The second is the zinc binding motif, which contains part of helix $\alpha 4$ and the following loop. The third region is part of the loop connecting helices $\alpha 4$ and $\alpha 5$. The fourth is residue Thr139 (Figure 4). These determinants cover four binding subsites of $\mathrm{BaP} 1$, one on the $\mathrm{N}$-terminal side of the catalytic center (S1) and three on the C-terminal side ( $\mathrm{S1}^{\prime}, \mathrm{S}^{\prime}$, and $\mathrm{S}^{\prime}$ ) (Figure 1B).

The first segment (Asn106-Thr107-Ile108-Gly109-Arg110) contributes to all peptide-binding subsites of $\mathrm{BaP} 1$. Thr107 and Arg110 are involved in forming subsite S1, Thr107 and Ile108 in subsite S1', Asn106 and Thr107 in subsite S2', and Asn106 and Ile108 in subsite $\mathrm{S}^{\prime}$. As the first segment is part of strand $\beta 4$, hydrogen bonding is dominated by backbone interactions, with bonds formed between the main chain $\mathrm{O}$ atom of Asn106 and the $\mathrm{N}$ atom of Myl4, between the $\mathrm{N}$ atom of Ile 108 and the $\mathrm{O}$ atom of Byl2, and between the $\mathrm{O}$ atom of Gly109 and the amide nitrogen of Hydl (Figure 4). These main chain interactions are the reason for the observed $1.4 \AA$ shift of the loop segment toward the inhibitor upon binding. Additionally, there are side chain hydrogen bonds between the $\mathrm{O} \delta$ atom of Asn106 and the $\mathrm{N}$ atom of Myl4 as well as between the $\mathrm{O} \gamma$ atom of Thr107 and the $\mathrm{O}$ atom of P1. Whereas the Thr107 side chain only slightly changes its orientation, the Asn106 side chain, which is solvent-exposed in the native structure, now points toward the inhibitor (Figure 4). Ile108 is part of the entrance of the $\mathrm{Sl}^{\prime}$ subsite tunnel, which is known for its high hydrophobicity in all SVMPs and is likely to be responsible for substrate specificity $(4,24)$. Its $\mathrm{C} \gamma$ and $\mathrm{C} \delta$ atoms are within van der Waals contact distance of the $\mathrm{C}$ atoms of $\mathrm{P}^{\prime}(3.8 \AA)$. Thr139 is also part of the $\mathrm{Sl}^{\prime}$ subsite tunnel entrance, and its $\mathrm{C} \gamma$ atom, as well as that of Thr107, is within hydrophobic contact distance of the inhibitor's $\mathrm{P1}^{\prime}$ residue (Figure 4).

A rarely seen type of inhibitor-enzyme interaction is observed between the Arg110 side chain (subsite S1) and the P1 residue of the peptidomimetic scaffold, as they form a cation $-\pi$ interaction. The delocalized positive charge of the arginine side chain interacts with the $\pi$-system of the thiazole ring. In the native structure of $\mathrm{BaP} 1, \mathrm{Arg} 110$ extends into the bulk solvent, hence increasing the height of the docking site wall (Figure 1B). Interestingly, in the $\mathrm{BaP} 1 \cdot$ inhibitor complex, it is flipped toward helix $\alpha 3$, forming a hydrogen bond with the Ser72 side chain (not shown) and placing the positive charge within contact distance of the ring of P1 (Figure 4). The observed distances (3.5-3.7 $\AA$ ) between N $\eta$ and $\mathrm{N} \varepsilon$ atoms of $\operatorname{Arg} 110$ and the plane of the aromatic thiazole ring of $\mathrm{P} 1$ are close to the energetically most favorable distance 


\begin{tabular}{|c|c|c|c|c|}
\hline & I & II & III & IV \\
\hline space group & $P 2_{1} 2_{1} 2_{1}$ & $P 2_{1} 2_{1} 2_{1}$ & $P 2_{1} 2_{1} 2_{1}$ & $P 2_{1} 2_{1} 2_{1}$ \\
\hline wavelength $(\AA)$ & 0.91841 & 0.91841 & 0.91841 & 0.91841 \\
\hline resolution range $(\AA)^{a}$ & $20.0-1.14(1.21-1.14)$ & $19.3-1.46(1.55-1.46)$ & $20.0-1.05(1.11-1.05)$ & $20.0-1.08(1.11-1.08)$ \\
\hline cell parameters $a, b, c(\AA)$ & $38.1,59.6,82.7$ & $38.0,59.5,81.8$ & $37.9,59.8,83.3$ & $37.9,59.8,83.1$ \\
\hline no. of unique reflections ${ }^{a}$ & $68715(10292)$ & $32970(4755)$ & $84722(10797)$ & $77288(4106)$ \\
\hline completeness $(\%)^{a}$ & $98.7(93.0)$ & $98.4(90.1)$ & $95.0(75.9)$ & $94.6(64.6)$ \\
\hline multiplicity of observations & 6.9 & 7.0 & 7.6 & 7.4 \\
\hline$I / \sigma(I)^{a}$ & $18.8(5.4)$ & $21.9(6.7)$ & $25.2(7.8)$ & $17.6(4.2)$ \\
\hline$R_{\text {meas }}(\%)^{a}$ & $7.9(38.0)$ & $6.7(28.0)$ & $5.6(24.1)$ & $7.2(38.1)$ \\
\hline$R_{\mathrm{sym}}(\%)^{a}$ & $7.3(34.4)$ & $6.2(25.4)$ & $5.2(21.7)$ & $6.7(33.4)$ \\
\hline Wilson $B\left(\AA^{2}\right)$ & 9.0 & 8.7 & 10.1 & 8.7 \\
\hline$V_{\mathrm{M}}\left(\AA^{3} \mathrm{Da}^{-1}\right)$ & 2.07 & 2.03 & 2.08 & 2.07 \\
\hline solvent content $(\%)$ & 40.5 & 40.0 & 40.8 & 40.7 \\
\hline
\end{tabular}

${ }^{a}$ Data in the highest-resolution shell are given in parentheses.

Table 2: Refinement and Model Quality

\begin{tabular}{|c|c|c|c|c|}
\hline & I & II & III & IV \\
\hline resolution range $(\AA)$ & $18.2-1.14$ & $17.8-1.46$ & $19.7-1.05$ & $18.2-1.08$ \\
\hline no. of unique reflections & 66242 & 32627 & 82161 & 74965 \\
\hline no. of reflections in test set ${ }^{a}$ & $2403(3.5)$ & $1631(5.0)$ & $2542(3.0)$ & $2318(3.0)$ \\
\hline$R_{\text {cryst }}(\%)^{b}$ & 13.0 & 14.8 & 11.7 & 11.8 \\
\hline$R_{\text {free }}(\%)^{b}$ & 16.0 & 17.8 & 14.4 & 14.3 \\
\hline \multicolumn{5}{|l|}{ no. of atoms } \\
\hline protein & 1747 & 1657 & 1698 & 1702 \\
\hline water molecules & 345 & 376 & 391 & 370 \\
\hline ligands & 51 & 37 & 37 & 42 \\
\hline total & 2143 & 2070 & 2126 & 2114 \\
\hline \multicolumn{5}{|l|}{$B$-factor $\left(\AA^{2}\right)$} \\
\hline main chain & 5.6 & 6.2 & 5.3 & 6.0 \\
\hline side chain & 8.0 & 9.0 & 8.1 & 8.7 \\
\hline ligands & 10.3 & 8.2 & 8.2 & 8.0 \\
\hline water molecules & 20.4 & 22.2 & 19.8 & 20.5 \\
\hline total & 9.1 & 10.3 & 9.1 & 9.8 \\
\hline \multicolumn{5}{|l|}{ rms deviations $^{b}$} \\
\hline bond lengths $(\AA)$ & 0.016 & 0.018 & 0.017 & 0.017 \\
\hline bond angles (deg) & 1.776 & 1.861 & 1.793 & 1.748 \\
\hline \multicolumn{5}{|l|}{ Ramachandran statistics $(\%)^{c}$} \\
\hline favored region & 98.0 & 98.5 & 98.5 & 99.0 \\
\hline allowed region & 2.0 & 1.5 & 1.5 & 1.0 \\
\hline outlier region & 0.0 & 0.0 & 0.0 & 0.0 \\
\hline
\end{tabular}

${ }^{a}$ The percentage of reflections in the test set is given in parentheses. ${ }^{b} R$-Factors and rms deviations after the last refinement step with REFMAC (44). ${ }^{c}$ Ramachandran statistics calculated with RAMPAGE (53).

(4.0 $\mathrm{A}$ ) for this kind of interaction. The P1 residue is also fixed by a hydrophobic interaction with the $\mathrm{C} \gamma$ atom (Figure 4).

The only amino acid of the zinc binding motif (ranging from His142 to Asp153) involved in BaP1 subsite building is His142. It is also part of the entrance of the $\mathrm{S1}^{\prime}$ pocket and thus interacting with residue $\mathrm{P}^{\prime}(\mathrm{C} \gamma$ and $\mathrm{C} \varepsilon$ atoms). On its other side ( $\mathrm{N} \varepsilon 2$ atom), it is involved in coordination of the zinc ion (Figure 4). As has been observed in the majority of 4-fold zinc coordination systems in enzymes, the zinc ion is tetrahedrally coordinated by the $\mathrm{N} \varepsilon 2$ atoms of three histidines (His142, His146, and His152) and a catalytic water molecule in the noncomplexed $\mathrm{BaP} 1$ structure (Figure 5A) $(24,50)$. In contrast, the zinc ion in the inhibitor complex is 5 -fold coordinated, with nearly perfect square pyramidal coordination geometry (Figure $5 \mathrm{~B}$ ). The $\mathrm{N} \varepsilon 2$ atoms of His 146 and His152 and both oxygens of Hyd 1 form the basal plane of the pyramid, and the Ne2 atom of His142 is the apex. The zinc ion is slightly displaced toward the apex, out of the plane of the basal atoms. Compared with the more common trigonal bipyramidal geometry, this square pyramidal arrangement is rarely observed in five-coordinate enzymatic zinc centers ( $26 \%$ vs $74 \%)(50)$.

The binding of the inhibitor results in the displacement of several water molecules at the docking site of native $\mathrm{BaP} 1$, i.e., the catalytic Wat67. Wat67 has been proposed to play a critical role as the catalytic nucleophile in SVMPs, attacking the scissile peptide bond after polarization by the highly conserved Glu143 (24). Only two water molecules, Wat7 and Wat13 deep inside the $\mathrm{Sl}^{\prime}$ pocket, were found near the active site in the inhibitor complex. Wat7 interacts with the backbone $\mathrm{O}$ atoms of Ile165 and Ala167 and Wat13 with the backbone $\mathrm{N}$ and $\mathrm{O}$ atoms of Ser171 as well as with the Ser163 side chain. Instead of coordinating the catalytic water molecule, Glu143 rotates toward the hydroxamate group ( $\mathrm{Hyd} 1)$ of the inhibitor and forms hydrogen bonds with it (Figure 4). The glycerol $\mathrm{O} 3$ atom is detected close to the original position of another water molecule in the native structure (Wat69).

Backbone atoms of the last segment (Ser168-Val169-Leu170) are the main part of the $\mathrm{S1}^{\prime}$ and $\mathrm{S} 2^{\prime}$ subsites of $\mathrm{BaP} 1$, interacting with the inhibitor scaffold via two hydrogen bonds. The first is formed by the backbone $\mathrm{O}$ atom of Ser168 with the backbone $\mathrm{N}$ atom of Byl3 and the second between the $\mathrm{O}$ atom of Byl 3 with the main chain $\mathrm{N}$ atom of Leu170. Presumably, these interactions force this loop segment on a $1.3 \AA$ shift toward the inhibitor upon binding. This could also be the reason for the rotamer change of the Ser168 side chain, although it is not directly involved in inhibitor binding (Figure 4). Besides the hydrogen bonds, almost all main chain atoms of the loop are within van der Waals contact distance of the inhibitor $\mathrm{P}^{\prime}$ residue. Additionally, the side chain atoms of Val169 are possible interaction partners for the $\mathrm{P} 2^{\prime}$ inhibitor residue, which points outside the cleft toward the bulk solvent. The $\mathrm{C} \gamma$ atoms of Val169 are located close (3.8 and $4.2 \AA$ ) to one of the methyl groups of $\mathrm{P} 2^{\prime}$, which probably imposes the Val167 side chain on a $90^{\circ}$ rotation (Figure 4). Because the side chain of Leu170 is positioned alongside the binding site cleft, it interacts with the $\mathrm{P}^{\prime}$ residue as well as with the $\mathrm{P} 3^{\prime}$ residue and is part of two different subsites $\left(\mathrm{Sl}^{\prime}\right.$ and $\left.\mathrm{S}^{\prime}\right)$.

Overall, inhibitor binding affects only the substrate binding pocket, while the rest of the protease is unperturbed. Inhibitor binding causes the pocket-flanking loops to shift inward by $2.7 \AA$, narrowing the substrate binding cleft. The most obvious differences occur at residues Asn106, Arg110, and Val169, which 
Lingott et al.
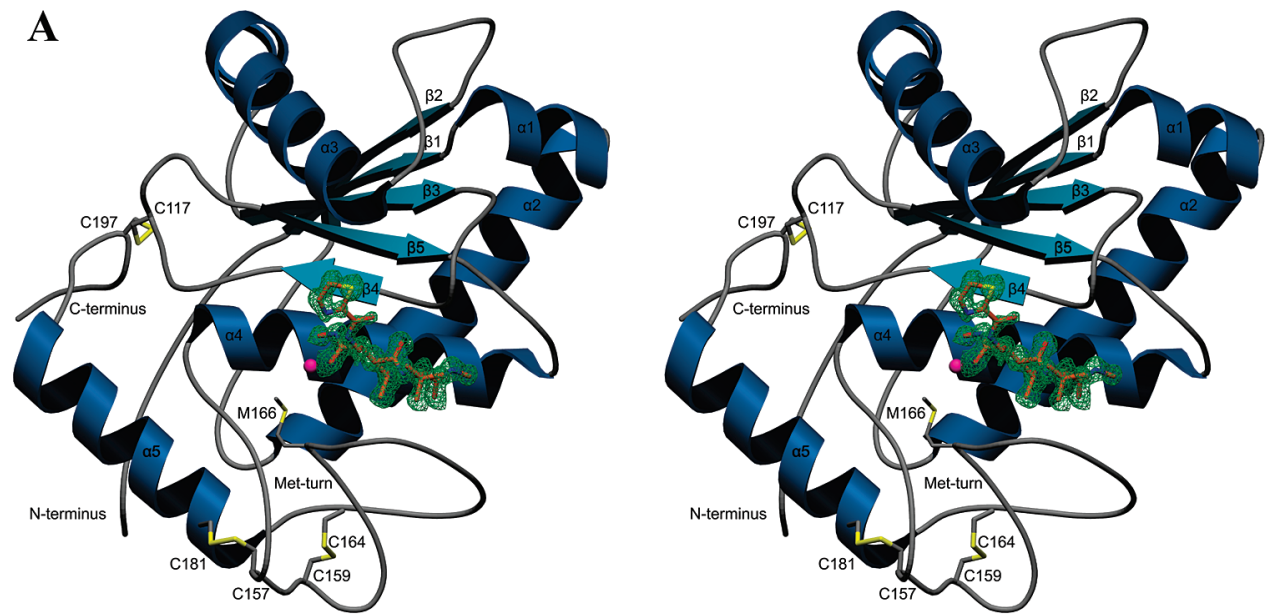

B
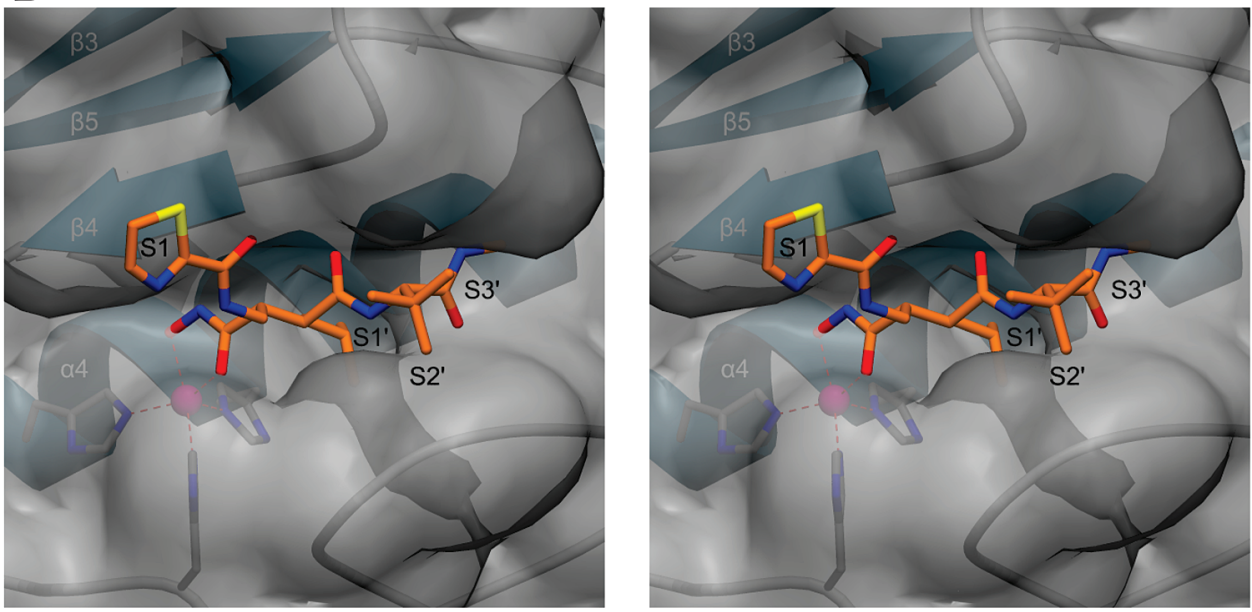

FIgURE 1: Stereoviews of the inhibitor bound to the snake venom metalloproteinase BaP1. (A) Ribbon plot of BaP1 with the initial $\left(F_{\mathrm{o}}-F_{\mathrm{c}}\right)$ electron density map of the inhibitor at a contour level of $3 \sigma$. The final model of the inhibitor is drawn into the density. The six cysteine residues involved in disulfide bonding and the conserved Met turn with the methionine residue are indicated for reference. The catalytic zinc ion is shown as a magenta sphere. (B) Inhibitor sitting in the shallow binding cleft of BaP1 represented by its molecular surface. The N-terminus of enzyme-bound substrate would be located on the left and the C-terminus on the right. The zinc ion, the coordinating histidine residues, and the ribbon plot of BaP1 are shown beneath the surface. Involved binding subsites of $\mathrm{BaP} 1$ are denoted with black labels and secondary structure elements with white labels.

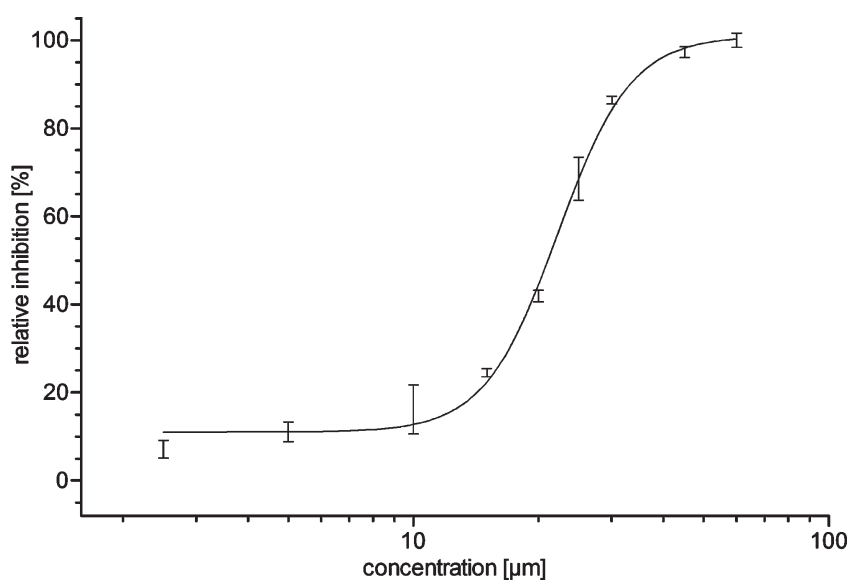

FIGURE 2: Inhibitory effect of the hydroxamate derivative on the proteolytic activity of BaP1. Sigmoidal dose-response curve as a semilog plot of the inhibitor concentration and the relative inhibition of the proteolytic activity of BaP1. Activity testing was determined on the basis of unselective cleavage of azocasein. Statistical analysis and nonlinear regression were performed with Prism 5.0 (GraphPad Software, Inc.) and led to an $\mathrm{IC}_{50}$ value of $22.2 \mu \mathrm{M}(95 \%$ confidence interval, $21.5-23.0 \mu \mathrm{M})$. Data are expressed as means \pm standard deviations of the relative inhibition.

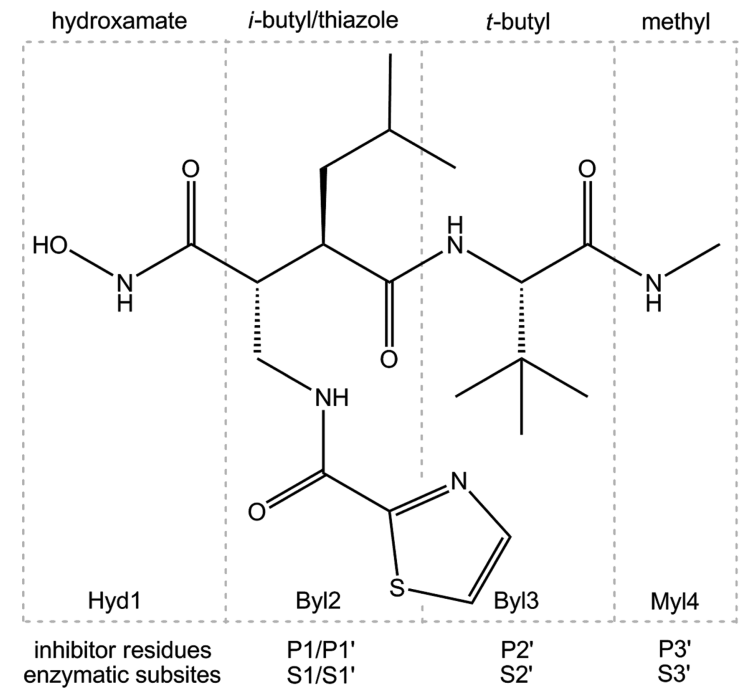

FIGURE 3: Schematic structure of the peptidomimetic inhibitor. Its functional groups (hydroxamate, isobutyl, tert-butyl, and methyl) are used for abbreviation and fragmentation of the scaffold (Hyd1, Byl2, Byl3, and Myl4). The $N$-methylthiazole-2-carboxamide group of Byl2 is shortened to thiazole. Inhibitor residues $\mathrm{P} 1-\mathrm{P} 3^{\prime}$ and enzymatic subsites involved in binding are indicated. 

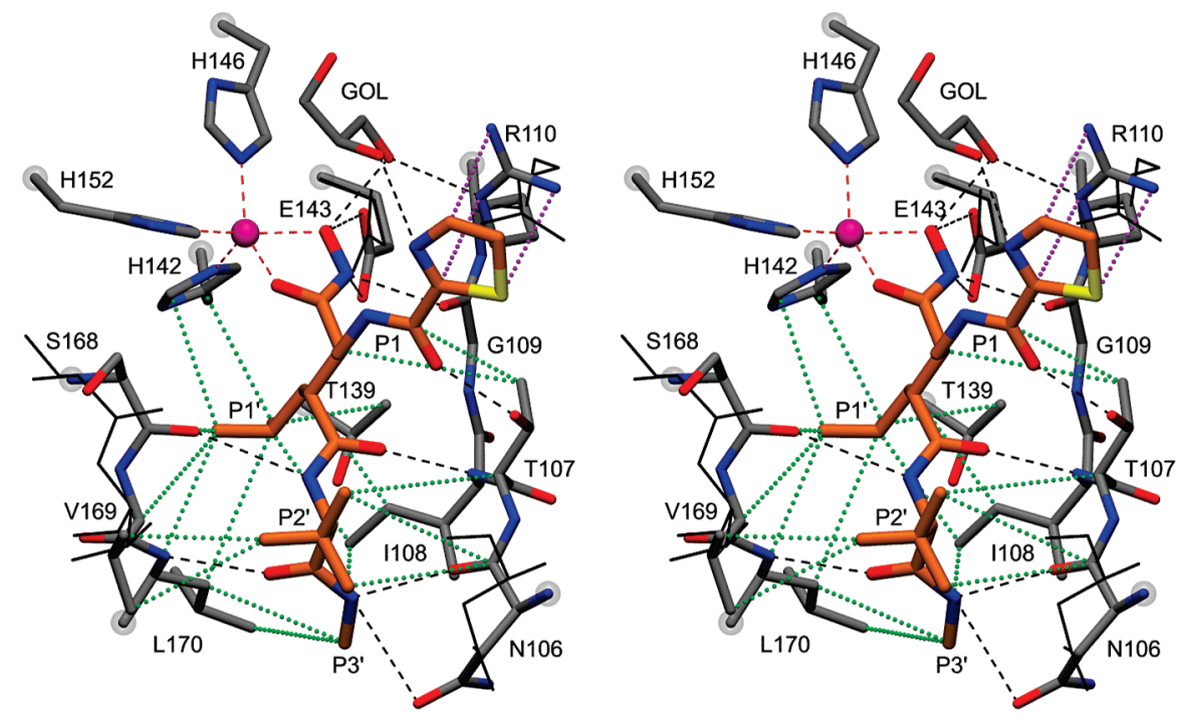

FIgURE 4: Stereoview of the BaP1 active site with the bound inhibitor as a stick model. Carbon atoms are colored gray (BaP1) and orange (inhibitor), oxygen atoms red, nitrogen atoms blue, and sulfur atoms yellow, and the catalytical zinc ion is shown as a magenta sphere. Important interactions are indicated as follows: hydrogen bonds as black dashed lines, zinc coordination as magenta dashed lines, hydrophobic contacts as green dots, and cation $-\pi$ interaction as purple dots. All enzymatic and inhibitor residues are indicated, and the complexed glycerol molecule is labeled with GOL. Side chains of Asp106, Arg110, Glu143, Ser168, and Val169 of noncomplexed BaP1 are shown as black sticks.

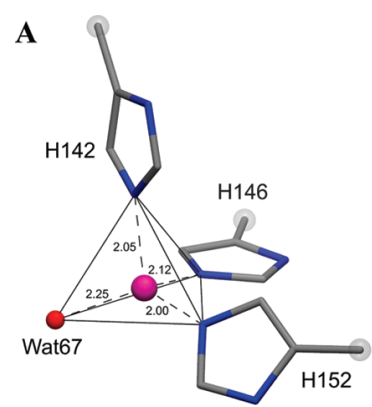

B

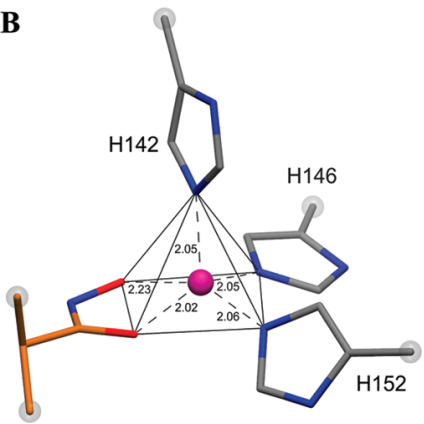

FIGURE 5: Zinc coordination sphere of noncomplexed and inhibitorbound BaP1. (A) Noncomplexed BaP1. The zinc ion is coordinated tetrahedrally by the $\mathrm{N} \varepsilon 2$ atoms of three histidine residues $(\mathrm{H} 142$, H146, and H152) and the oxygen atom of the catalytical water molecule (Wat67). Carbon atoms are shown as gray and nitrogen atoms as blue sticks. The water molecule is represented as a red sphere and the zinc ion as a magenta sphere. Coordination distances are all given in angstroms and taken from PDB entry 1ND1 (24). (B) BaP1 with the complexed inhibitor (only Hyd 1 is depicted). The zinc ion is coordinated in a nearly perfect square-based pyramidal geometry by the $\mathrm{N} \varepsilon 2$ atoms of three histidine residues (H142, H146, and H152) and both oxygen atoms of Hyd1. The latter atoms and those of His 146 and His 152 form the basal plane of the pyramid, and the $\mathrm{N} \varepsilon 2$ atom of His 142 is the apex. Carbon atoms are shown as gray, nitrogen atoms as blue, and oxygen atoms as red sticks, and the zinc ion is represented as a magenta sphere. Coordination distances are given in angstroms and represent the average values of all four presented structures. bind the inhibitor directly and change rotamers (Figure 4). This flexibility at the binding pocket is in agreement with the broad substrate specificity seen in SVMPs, making BaP1 well suited to cleave a variety of peptides in the extracellular matrix.

Zinc Coordination Geometry and $p H$ Dependency of Proteolytic Activity. The catalytic zinc ion in $\mathrm{BaP} 1$ changes its coordination geometry from four-coordinate tetrahedral in the unliganded native state to five-coordinate square pyramidal in the inhibitor complex (Figure 5 and Table 3). Such an expansion is expected to result in longer zinc coordination distances (50). However, this is only the case for His152 (2.063 \pm $0.009 \AA$ for the $\mathrm{Zn}-\mathrm{N} \varepsilon 2$ distance vs $2.0 \AA$ in the native structure). The corresponding distance to His 142 remains equal $(2.048 \pm 0.011 \AA$ vs $2.05 \AA)$, while the one to His146 even decreases $(2.048 \pm 0.005 \AA$ vs $2.12 \AA)$.

For SVMPs, it has been suggested that proteolytic activity depends on the protonation state of the zinc-coordinating histidine residues on one hand and on the polarization capacity of the glutamate residue on the other (4). In a study on acutolysin $\mathrm{C}$ using X-ray absorption near-edge structure spectroscopy (XANES) at different $\mathrm{pH}$ values, it was shown that with a change in $\mathrm{pH}$ from 8.0 to 3.0 the overall arrangement of the catalytic zinc ion remained tetrahedral, but the zinc coordination distances and the distance between the glutamate and the active site water molecule increased (51). In a similar vein, $\mathrm{pH}$-dependent changes in the protonation state of the active site histidines have been proposed to cause a change in zinc coordination distances (35). To address this question, we performed an additional step of refinement with REFMAC in which all metal-ligand restraints had been removed. Subsequent analysis of the $\mathrm{BaP} 1 \cdot$ inhibitor complex at four different $\mathrm{pH}$ values between 4.6 and 8.0 showed no detectable trend in zinc coordination (Table 3). All three histidines feature quite similar average Zn-N distances: $2.048 \pm 0.011 \AA$ (His142 N $\varepsilon 2$ atom), $2.048 \pm$ $0.005 \AA$ (His146 N $\varepsilon 2$ atom), and $2.063 \pm 0.009 \AA$ (His152 N $\varepsilon 2$ atom). Standard deviations are calculated between the four presented structures and are all within coordinate error $(0.025$ $\AA$ for data set IV). Likewise, there is no significant change in the 
Table 3: Coordination Distances ${ }^{a}$ at the Active Site of Complexed and Noncomplexed BaP1

\begin{tabular}{|c|c|c|c|c|c|}
\hline & I, $\mathrm{pH} 4.6$ & II, pH 6.5 & III, pH 7.5 & IV, $\mathrm{pH} 8.0$ & $\mathrm{BaP} 1,{ }^{b} \mathrm{pH} 9.0$ \\
\hline His $142(\mathrm{~N} \varepsilon 2)-\mathrm{Zn}^{2+}$ & 2.04 & 2.08 & 2.03 & 2.04 & 2.05 \\
\hline His $146(\mathrm{~N} \varepsilon 2)-\mathrm{Zn}^{2+}$ & 2.06 & 2.04 & 2.05 & 2.04 & 2.12 \\
\hline His $152(\mathrm{~N} \varepsilon 2)-\mathrm{Zn}^{2+}$ & 2.06 & 2.09 & 2.05 & 2.05 & 2.00 \\
\hline $\operatorname{Hyd} 1(\mathrm{OH})-\mathrm{Zn}^{2+}$ & 2.23 & 2.23 & 2.24 & 2.22 & - \\
\hline $\operatorname{Hyd} 1(\mathrm{O})-\mathrm{Zn}^{2+}$ & 2.01 & 2.03 & 2.01 & 2.02 & - \\
\hline Glu143(Oع1)-Hyd1(OH) & 2.62 & 2.54 & 2.61 & 2.67 & - \\
\hline Glu143(Oع2)-Hyd1(N) & 3.24 & 3.23 & 3.25 & 3.27 & - \\
\hline Wat67- $\mathrm{Zn}^{2+}$ & - & - & - & - & 2.25 \\
\hline Glu143(Oع1)-Wat69 & - & - & - & - & 2.86 \\
\hline Glu143(Oع2)-Wat67 & - & - & - & - & 3.01 \\
\hline
\end{tabular}

${ }^{a}$ All coordination distances are given in angstroms. ${ }^{b}$ Data taken from the noncomplexed structure of BaP1 (PDB entry 1ND1) (24).

distances between the zinc ion and the hydroxylic $\mathrm{Hyd} 1(\mathrm{OH})$ and carboxylic Hyd $1(\mathrm{O})$ oxygen atoms of the inhibitor $(2.230 \pm 0.004$ and $2.018 \pm 0.005 \AA$, respectively).

As mentioned above, it is generally accepted that the conserved glutamate residue plays an important role in acting as a general base during the reaction mechanism of metzincins (3). During the first step (addition), the catalytic water molecule is deprotonated, allowing its nucleophilic attack at the carbon atom of the peptide bond. This causes a tetrahedral reaction intermediate and a protonated glutamate residue. During the second step (elimination), the proton is transferred to the $\mathrm{N}$-terminus of the cleaved substrate. Presumably, the proteolytic reaction mechanism is trapped by the peptidomimetic inhibitor in the transition state of the reaction. Although the protonation state of the glutamate residue cannot be determined through a simple X-ray experiment, one may reasonably assume so, because both oxygen atoms of the glutamate residue are found to be within hydrogen bonding distance of the hydroxamate group of the inhibitor (Table 3). Unlike the decreasing distances between the glutamate residue and the catalytic water molecule in nonliganded SVMPs, no evidence exists for such a change in the $\mathrm{BaP} 1$-inhibitor complexes. No significant variation of the corresponding Glu143 $(\mathrm{O} \varepsilon 1)-\mathrm{Hyd} 1(\mathrm{OH})$ and $\mathrm{Glu} 143(\mathrm{O} \varepsilon 2)-\mathrm{Hyd} 1(\mathrm{~N})$ distances can be observed at the different $\mathrm{pH}$ values (Table 3 ). In contrast to proteolytic activity, this suggests that inhibitor binding does not seem to be dependent on $\mathrm{pH}$. Nevertheless, conclusions concerning atomic distances should be regarded critically, even though the estimated overall coordinate errors of the present highresolution structures are lower than those of previously available low-resolution structures (1.8-2.2 $⿱$ ) (35).

Another possible mechanism for $\mathrm{pH}$-dependent modulation of proteolytic activity is a more widespread change in enzyme structure (52). For the BaP1·inhibitor complex, there are only a few side chain and loop shifts, mainly at the protein surface and the active site, but no significant conformational changes in the peptide backbone at different $\mathrm{pH}$ values can be observed. Structural alignments lead to $\mathrm{C} \alpha$ rms deviations between 0.05 and $0.20 \AA$ in pairwise comparisons. Thus, the structures can be regarded as the same, and our high-resolution analysis does not support the idea of wide-range conformational shifts causing a change in activity.

Flexible Loop Region and Its Importance for Hemorrhagic Activity. Several investigations have correlated surface characteristics and structural variations within the family of SVMPs, especially at a loop region comprising residues $153-$ 176, with their hemorrhagic potential (24-26). This loop surrounds the active site and contains the highly conserved Met turn

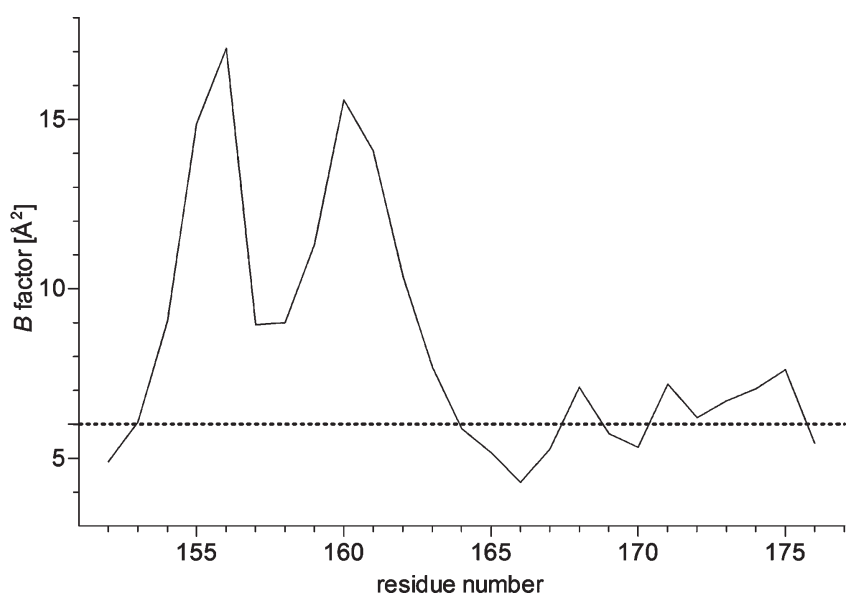

Figure 6: $B$-Factor plot of the flexible loop region (residues 152176). For reference, the average $B$-factor of all $\mathrm{BaP} 1 \mathrm{C} \alpha$ atoms $\left(6.01 \AA^{2}\right)$ is indicated as a dotted line.

$\left(\mathrm{C}_{164} \mathrm{I}_{165} \mathrm{M}_{166}\right)$. It was proposed that this loop, by virtue of its surface localization and its proximity to the active site, mediates extracellular matrix substrate binding and thereby influences the hemorrhagic potential of the enzymes. In our analysis of $\mathrm{BaP} 1$, the first part of the loop (residues 154-162) shows high flexibility, with considerably higher $B$-factors (values for main and side chain atoms of 12.8 and $15.2 \AA^{2}$, respectively) compared to the average values of the whole protein $(6.0$ and $8.7 \AA^{2}$, respectively). The last four residues in this segment (159-162) are in a double conformation in all four BaP1 -inhibitor structures, representing a superposition of closed and open conformations. In the closed conformation, this loop segment packs against residues 175-177 through backbone interactions, while it extends into the bulk sovent in the open conformation. In contrast to this highly flexible segment, the remainder of the loop region on the $\mathrm{C}$-terminal side of the Met turn is rigid again (6.6 and $9.1 \AA^{2}$, residues 167-176) (Figure 6). A comparison of the $B$-factor distribution of identical residues in the noncomplexed form of $\mathrm{BaP} 1$ shows the same tendencies in flexibility by the unchanged rigidity of the active site residues (data not shown).

This observation displays the flexibility of the loop and argues for its functional relevance. As the catalytic center and other parts involved in proteolytic activity, like the conserved Met turn, are not affected by the loop's conformational change, one can assume that the protein is capable of varying its surface properties without influencing its proteolytic activity. Thereby, it would be enabled to dock to different kind of substrates in the extracellular 
matrix and nevertheless restore its functionality as a peptidecleaving enzyme.

\section{CONCLUSIONS}

Metzincins play an important role in many diseases affecting humans. However, effective and selective drugs against metalloproteinases are still rare. There is no neutralizing drug against the local pathological effects caused by snake bites, and in spite of massive research, only a few inhibitors against human ADAMs and MMPs have passed clinical trials. In the majority of cases, these small molecule inhibitors contain a zinc-binding group (ZBG), a peptide backbone or peptidomimetic scaffold, and suitable side chains that bind to the enzymatic subsites. Because metalloproteinases often cleave a wide range of targets, they are quite flexible in binding peptides and display broad substrate specificity. Thus, developing highly selective metalloproteinase inhibitors still poses one of the main challenges in the search for successful clinical candidates. To that end, three-dimensional structures of metalloproteinase-inhibitor complexes present crucial information about the structural determinants of substrate selectivity.

The $\mathrm{BaP} 1 \cdot$ inhibitor complex reported here contributes to metalloproteinase crystal structures with one of the best resolution ranges ever obtained. Despite the moderate $\mathrm{IC}_{50}$ value of the inhibitor, the high-resolution structure is an excellent starting point for further optimization. The atomic detail offers insight into inhibitor binding and the structural determinants for proteolytic activity. In particular, the deep tunnel-like $\mathrm{Sl}^{\prime}$ subpocket of $\mathrm{BaP} 1$ suggests the inhibitor might be improved by using a longer residue at the $\mathrm{P}^{\prime}$ position. Because of the poor bioavailability of hydroxamate derivatives, a change of the ZBG might also be considered.

The finding about the flexible loop region, which is possibly correlating with hemorrhagic activity, has a great impact on further studies, as to date only little about hemorrhagic activity and its structural and sequential dependencies is known. More detailed insight into structural requirements for the hemorrhagic potential of snake venoms will be helpful for the local therapy of snake venom poisonings. A comparison of the BaP1 inhibitor complex with human metalloproteinases reveals similarities in terms of not only the overall protein structure but also specifically the shape and properties of the binding site. Accordingly, the reported inhibitor, which was primarily designed as a TACE inhibitor, is significantly blocking the activity of BaP1, thus confirming the similarities in the arrangement and mode of action of the catalytic domains. This highlights $\mathrm{BaP} 1$, whose isolation from snake venom is straightforward, as a model system not only for SVMPs but also for relevant medicinal, human metalloproteinase targets.

\section{ACKNOWLEDGMENT}

We thank Georg Zocher of the BESSY supporting team for guidance during data collection and Roche Pharmaceutics for providing the inhibitor. Daniel P. Kloer's careful reading of the manuscript and his helpful suggestions are gratefully acknowledged.

\section{REFERENCES}

(1) Bjarnason, J. B., and Fox, J. W. (1995) Snake-venom metalloendopeptidases: Reprolysins. Methods Enzymol. 248, 345-368.
(2) Bode, W., Gomis-Ruth, F. X., and Stocker, W. (1993) Astacins, serralysins, snake-venom and matrix metalloproteinases exhibit identical zinc-binding environments (HExxHxxGxxH and Metturn) and topologies and should be grouped into a common family, the metzincins. FEBS Lett. 331, 134-140.

(3) Gomis-Ruth, F. X. (2003) Structural aspects of the metzincin clan of metalloendopeptidases. Mol. Biotechnol. 24, 157-202.

(4) Ramos, O. H. P., and Selistre-De-Araujo, H. S. (2006) Snake venom metalloproteases: Structure and function of catalytic and disintegrin domains. Comp. Biochem. Phys., Part C: Pharmacol., Toxicol. Endocrinol. 142, 328-346.

(5) Stocker, W., Grams, F., Baumann, U., Reinemer, P., Gomis-Ruth, F. X., Mckay, D. B., and Bode, W. (1995) The metzincins: Topological and sequential relations between the astacins, adamalysins, serralysins, and matrixins (collagenases) define a superfamily of zinc-peptidases. Protein Sci. 4, 823-840.

(6) Gomis-Ruth, F. X. (2009) Catalytic domain architecture of metzincin metalloproteases. J. Biol. Chem. 284, 15353-15357.

(7) Gomis-Ruth, F. X., Kress, L. F., and Bode, W. (1993) First structure of a snake venom metalloproteinase: A prototype for matrix metalloproteinases/collagenases. EMBO J. 12, 4151-4157.

(8) Moss, M. L., Sklair-Tavron, L., and Nudelman, R. (2008) Drug insight: Tumor necrosis factor-converting enzyme as a pharmaceutical target for rheumatoid arthritis. Nat. Clin. Pr. Rheumatol. 4 , 300-309.

(9) Takeda, S. (2009) Three-dimensional domain architecture of the ADAM family proteinases. Semin. Cell Dev. Biol. 20, 146-152.

(10) Wolfsberg, T. G., Bazan, J. F., Blobel, C. P., Myles, D. G., Primakoff, P., and White, J. M. (1993) The precursor region of a protein active in sperm-egg fusion contains a metalloprotease and a disintegrin domain: Structural, functional, and evolutionary implications. Proc. Natl. Acad. Sci. U.S.A. 90, 10783-10787.

(11) Seals, D. F., and Courtneidge, S. A. (2003) The ADAMs family of metalloproteases: Multidomain proteins with multiple functions. Genes Dev. 17, 7-30.

(12) Kenny, P. A. (2007) TACE: A new target in epidermal growth factor receptor dependent tumors. Differentiation 75, 800-808.

(13) White, J. M. (2003) ADAMs: Modulators of cell-cell and cell-matrix interactions. Curr. Opin. Cell Biol. 15, 598-606.

(14) World Health Organization (2007) Rabies and envenomings: A neglected public health issue. World Health Organization, Geneva.

(15) Gutiérrez, J. M., León, G., Rojas, G., Lomonte, B., Rucavado, A., and Chaves, F. (1998) Neutralization of local tissue damage induced by Bothrops asper (Terciopelo) snake venom. Toxicon 36, 1529-1538.

(16) Gutiérrez, J. M., and Rucavado, A. (2000) Snake venom metalloproteinases: Their role in the pathogenesis of local tissue damage. Biochimie 82, 841-850.

(17) Bjarnason, J. B., and Fox, J. W. (1994) Hemorrhagic metalloproteinases from snake-venoms. Pharmacol. Ther. 62, 325-372.

(18) Gutiérrez, J. M., Romero, M., Díaz, C., Borkow, G., and Ovadia, M. (1995) Isolation and characterization of a metalloproteinase with weak hemorrhagic activity from the venom of the snake Bothrops asper (Terciopelo). Toxicon 33, 19-29.

(19) Rucavado, A., Núñez, J., and Gutiérrez, J. M. (1998) Blister formation and skin damage induced by BaP1, a haemorrhagic metalloproteinase from the venom of the snake Bothrops asper. Int. J. Exp. Pathol. 79, 245-254.

(20) Fox, J. W., and Serrano, S. M. T. (2008) Insights into and speculations about snake venom metalloproteinase (SVMP) synthesis, folding and disulfide bond formation and their contribution to venom complexity. FEBS J. 275, 3016-3030.

(21) Gutiérrez, J. M. (1995) Clinical toxicology of snakebite in Central America. In Clinical Toxicology of Animal Venoms and Poisons (Meier, J., and White, J., Eds.) pp 646-665, CRC Press, Boca Raton, FL.

(22) Fernandes, C. M., Zamuner, S. R., Zuliani, J. P., Rucavado, A., Gutiérrez, J. M., and Teixeira, C. D. P. (2006) Inflammatory effects of $\mathrm{BaPl}$ a metalloproteinase isolated from Bothrops asper snake venom: Leukocyte recruitment and release of cytokines. Toxicon 47, 549-559.

(23) Rucavado, A., Lomonte, B., Ovadia, M., and Gutiérrez, J. M. (1995) Local tissue damage induced by BaP1, a metalloproteinase isolated from Bothrops asper (Terciopelo) snake venom. Exp. Mol. Pathol. 63, 186-199.

(24) Watanabe, L., Shannon, J. D., Valente, R. H., Rucavado, A., ape-Giron, A., Kamiguti, A. S., Theakston, R. D. G., Fox, J. W., Gutiérrez, J. M., and Arni, R. K. (2003) Amino acid sequence and crystal structure of $\mathrm{BaP1}$, a metalloproteinase from Bothrops asper snake venom that exerts multiple tissue-damaging activities. Protein Sci. 12, 2273-2281. 
(25) Gong, W. M., Zhu, X. Y., Liu, S. J., Teng, M. K., and Niu, L. W. (1998) Crystal structures of acutolysin A, a three-disulfide hemorrhagic zinc metalloproteinase from the snake venom of Agkistrodon acutus. J. Mol. Biol. 283, 657-668.

(26) Ramos, O. H. P., and Selistre-De-Araujo, H. S. (2004) Comparative analysis of the catalytic domain of hemorrhagic and non-hemorrhagic snake venom metallopeptidases using bioinformatic tools. Toxicon 44, 529-538.

(27) Stocker, K. (1999) Use of snake venom proteins in medicine. Schweiz. Med. Wochenschr. 129, 205-216.

(28) Koh, D. C. I., Armugam, A., and Jeyaseelan, K. (2006) Snake venom components and their applications in biomedicine. Cell. Mol. Life Sci. 63, 3030-3041.

(29) Ondetti, M. A., Williams, N. J., Sabo, E. F., Pluscec, J., Weaver, E. R., and Kocy, O. (1971) Angiotensin-converting enzyme inhibitors from venom of Bothrops jararaca. Isolation, elucidation of structure, and synthesis. Biochemistry 10, 4033-4039.

(30) Botos, I., Scapozza, L., Zhang, D. C., Liotta, L. A., and Meyer, E. F. (1996) Batimastat, a potent matrix metalloproteinase inhibitor, exhibits an unexpected mode of binding. Proc. Natl. Acad. Sci. U. S.A. 93, 2749-2754.

(31) Cirilli, M., Gallina, C., Gavuzzo, E., Giordano, C., Gomisruth, F. X., Gorini, B., Kress, L. F., Mazza, F., Paradisi, M. P., Pochetti, G., and Politi, V. (1997) 2 angstrom X-ray structure of adamalysin II complexed with a peptide phosphonate inhibitor adopting a retrobinding mode. FEBS Lett. 418, 319-322.

(32) Gomis-Ruth, F. X., Meyer, E. F., Kress, L. F., and Politi, V. (1998) Structures of adamalysin II with peptidic inhibitors. Implications for the design of tumor necrosis factor $\alpha$ convertase inhibitors. Protein Sci. 7, 283-292.

(33) Huang, K. F., Chiou, S. H., Ko, T. P., and Wang, A. H. J. (2002) Determinants of the inhibition of a Taiwan habu venom metalloproteinase by its endogenous inhibitors revealed by X-ray crystallography and synthetic inhibitor analogues. Eur. J. Biochem. 269, 3047-3056.

(34) Lou, Z., Hou, J., Liang, X., Chen, J., Qiu, P., Liu, Y., Li, M., Rao, Z., and Yan, G. (2005) Crystal structure of a non-hemorrhagic fibrin (ogen)olytic metalloproteinase complexed with a novel natural tripeptide inhibitor from venom of Agkistrodon acutus. J. Struct. Biol. 152, 195-203.

(35) Zhu, X. Y., Teng, M. K., and Niu, L. W. (1999) Structure of acutolysin C, a haemorrhagic toxin from the venom of Agkistrodon acutus, providing further evidence for the mechanism of the $\mathrm{pH}-$ dependent proteolytic reaction of zinc metalloproteinases. Acta Crystallogr. D55, 1834-1841.

(36) Jacobsen, F. E., Lewis, J. A., and Cohen, S. M. (2007) The design of inhibitors for medicinally relevant metalloproteins. Chem. Med. Chem. 2, 152-171.

(37) Gomis-Ruth, F. X., Kress, L. F., Kellermann, J., Mayr, I., Lee, X., Huber, R., and Bode, W. (1994) Refined 2.0 angstrom X-ray crystal structure of the snake-venom zinc-endopeptidase adamalysin II: Primary and tertiary structure determination, refinement, molecular-structure and comparison with astacin, collagenase and thermolysin. J. Mol. Biol. 239, 513-544.
(38) Huang, K. F., Chiou, S. H., Ko, T. P., Yuann, J. M., and Wang, A. H. (2002) The $1.35 \AA$ structure of cadmium-substituted TM-3, a snake-venom metalloproteinase from Taiwan habu: Elucidation of a TNF $\alpha$-converting enzyme-like active-site structure with a distorted octahedral geometry of cadmium. Acta Crystallogr. D58, 11181128.

(39) Kumasaka, T., Yamamoto, M., Moriyama, H., Tanaka, N., Sato, M., Katsube, Y., Yamakawa, Y., OmoriSatoh, T., Iwanaga, S., and Ueki, T. (1996) Crystal structure of H-2-proteinase from the venom of Trimeresurus flavoviridis. J. Biochem. 119, 49-57.

(40) Zhang, D. C., Botos, I., Gomis-Ruth, F. X., Doll, R., Blood, C., Njoroge, F. G., Fox, J. W., Bode, W., and Meyer, E. F. (1994) Structural interaction of natural and synthetic inhibitors with the venom metalloproteinase, atrolysin-C (form-D). Proc. Natl. Acad. Sci. U.S.A. 91, 8447-8451.

(41) Laemmli, U. K. (1970) Cleavage of structural proteins during assembly of head of bacteriophage-T4. Nature 227, 680-685.

(42) Wang, W. J., Shih, C. H., and Huang, T. F. (2004) A novel P-I class metalloproteinase with broad substrate-cleaving activity, agkislysin, from Agkistrodon acutus venom. Biochem. Biophys. Res. Commun. $324,224-230$

(43) Kabsch, W. (1988) Evaluation of single-crystal X-ray-diffraction data from a position-sensitive detector. J. Appl. Crystallogr. 21, 916-924.

(44) Murshudov, G. N., Vagin, A. A., and Dodson, E. J. (1997) Refinement of macromolecular structures by the maximum-likelihood method. Acta Crystallogr. D53, 240-255.

(45) Emsley, P., and Cowtan, K. (2004) Coot: Model-building tools for molecular graphics. Acta Crystallogr. D60, 2126-2132.

(46) Schuttelkopf, A. W., and van Aalten, D. M. F. (2004) PRODRG: A tool for high-throughput crystallography of protein-ligand complexes. Acta Crystallogr. D60, 1355-1363.

(47) Lamzin, V. S., and Wilson, K. S. (1997) Automated refinement for protein crystallography. Methods Enzymol. 277, 269-305.

(48) Fenn, T. D., Ringe, D., and Petsko, G. A. (2003) POVScript +: A program for model and data visualization using persistence of vision ray-tracing. J. Appl. Crystallogr. 36, 944-947.

(49) Kraulis, P. J. (1991) Molscript: A program to produce both detailed and schematic plots of protein structures. J. Appl. Crystallogr. 24, 946-950.

(50) Alberts, I. L., Nadassy, K., and Wodak, S. J. (1998) Analysis of zinc binding sites in protein crystal structures. Protein Sci. 7, 1700-1716.

(51) Zhao, W., Chu, W. S., Li, S. J., Liu, Y. W., Gao, B., Niu, L. W., Teng, M. K., Benfatto, M., Hu, T. D., and Wu, Z. Y. (2007) X-ray absorption near edge structure study on acutolysin $\mathrm{C}$, a zincmetalloproteinase from Agkistrodon acutus venom: Insight into the acid-inactive mechanism. Spectrochim. Acta, Part B 62, 1246-1251.

(52) Xu, X. L., Liu, X. H., Wu, B., Liu, Y., Liu, W. Q., Xie, Y. S., and Liu, Q. L. (2004) Metal-ion- and $\mathrm{pH}$-induced conformational changes of acutolysin D from Agkistrodon acutus venom probed by fluorescent spectroscopy. Biopolymers 74, 336-344.

(53) Lovell, S. C., Davis, I. W., Adrendall, W. B., de Bakker, P. I. W., Word, J. M., Prisant, M. G., Richardson, J. S., and Richardson, D. C. (2003) Structure validation by $\mathrm{C} \alpha$ geometry: $\phi, \psi$ and $\mathrm{C} \beta$ deviation. Proteins 50, 437-450. 